\title{
Properties of Intermittent Bursts in Edge Plasma of LHD
}

\author{
Hayato TSUCHIYA, Tomohiro MORISAKI, Viatcheslav P. BUDAEV ${ }^{1)}$, Akio KOMORI, \\ Hiroshi YAMADA and LHD Experimental Group \\ National Institute for Fusion Science, Toki 509-5292, Japan \\ 1) Nuclear Fusion Institute, RRC Kurchatov Institute, 123182, Kurchatov Sq.1, Moscow, Russia
}

(Received 5 January 2010 / Accepted 16 March 2010)

\begin{abstract}
In edge plasma in the Large Helical Device (LHD), incoherent fluctuations with intermittent density bursts were observed with a two-dimensional lithium beam probe that can measure electron density and its fluctuation two-dimensionally. Statistical analysis using a probability denstiy function (PDF) showed that those bursts have the characteristics of blobs, which have been observed in tokamaks. Two-dimensional PDF analysis indicates that the blobs originate in the ergodic layer outside the last closed flux surface and move to the outer region. Wavelet analysis showed that the direction of blob propagation is not always outward but is sometimes in arbitrary directions. These characteristics may be a result of the complicated magnetic field structure of the heliotron configuration.
\end{abstract}

(c) 2010 The Japan Society of Plasma Science and Nuclear Fusion Research

Keywords: intermittent transport, blob, LHD, edge plasma

DOI: $10.1585 /$ pfr.5.S2078

\section{Introduction}

Edge fluctuation is important in energy and particle transport in the edge region of a torus plasma. In addition to coherent and turbulent fluctuations, interest has recently arisen in intermittent or burst-like phenomena such as edge localized modes (ELMs) and blobs [1-5], which cause large radial transport of particles from the scrape-off layer (SOL) to the vacuum vessel wall. In a reactor plasma, such phenomena may pose a critical problem since they not only cause plasma exhaust but also damage the vessel wall with their considerable heat load. Thus, it is critical to the establish techniques for controlling ELMs and blobs. However, the physical mechanism of the phenomena is not adequately explained [6-10], and experimental observations are few. Observations are difficult beacause ELMs and blobs can appear suddenly in any location and have no poloidal symmetries.

To observe such phenomena effectively, we have developed a two-dimensional(2D) thermal lithium beam probe (2D-LiBP) [11-14] to two-dimensionally measure the edge density and its fluctuations. In this paper, blob characteristics observed in the Large Helical Device (LHD) [15] with the 2D-LiBP are presented.

\section{Experimental Setup}

Figure 1 shows a schematic of the experimental setup. A sheet-shaped thermal lithium beam is injected on the poloidal plane from the bottom of the torus. The thickness of the beam in the toroidal direction at $Z=-1 \mathrm{~m}$ is $\sim 9 \mathrm{~cm}[14]$, which determines the spatial resolution in the

author's e-mail: tsuchiya.hayato@LHD.nifs.ac.jp toroidal direction. The $\mathrm{Li}$ I light $(670.8 \mathrm{~nm})$ due to electron impact excitation is detected by a charge-coupled device (CCD) to measure the density, and an optical fiber bundle coupled with photomultiplier tubes (PMTs) is used to measure the fluctuation, from the outer port on the equatorial plane through the interference filter. Red circles in Fig. 1 correspond to the observing position of each optical fiber on the poloidal cross section where the beam is injected. The numbered fibers are actually connected to PMTs. Thus eight channel signals were digitized at $1 \mathrm{MHz}$ in this experiment. The spatial resolution of the fiber optics is about $5 \mathrm{~cm}$ in the poloidal plane. The observing area is limited to the edge region where the magnetic field lines have an ergodic structure, as shown in Fig. 1, since the beam energy is too low to penetrate deep inside the plasma.

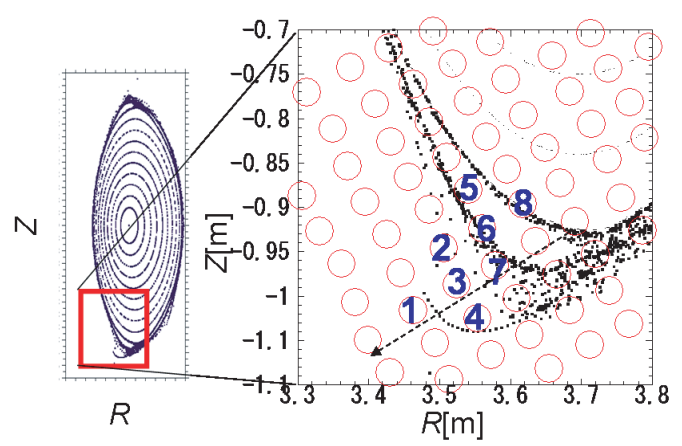

Fig. 1 Schematic drawing of 2D-LiBP system with observing position of each optical fiber for fluctuation measurement. Numbered fibers are those used in this experiment. 


\section{Results}

The LHD is a superconducting heliotron device with major and minor radii of $3.9 \mathrm{~m}$ and $0.6 \mathrm{~m}$, respectively. Experiments were performed with hydrogen plasma produced by electron cyclotron resonance heating. The lineaveraged electron density at the center was $\bar{n}_{\mathrm{e}} \leq 7.7 \times$ $10^{18} \mathrm{~m}^{-3}$. Fast Fourier transform (FFT) analysis revealed no coherent power peaks in the fluctuations. The broad spectra found by FFT analysis may indicate that the fluctuation in the ergodic region is turbulent. However, intermittent positive spikes indicating the blobs of dense plasma often appear in the time series signals of the outer channels, as shown in Fig. 2. Some spikes (marked with rectangles) seem to propagate from ch7 to ch2 through ch3, which suggests that particles are transported by the blobs in the same direction. From the time delay between channels, the velocity $V_{\mathrm{b}}$ of a blob can easily be derived, for example, $V_{\mathrm{b} 7 \rightarrow 3} \sim 1.0 \mathrm{~km} / \mathrm{s}, V_{\mathrm{b} 3 \rightarrow 2} \sim 2.5 \mathrm{~km} / \mathrm{s}$. Since the spatial resolution of the optical systems is $5 \mathrm{~cm}$, the burst size is believed to be less than $5 \mathrm{~cm}$.

FFT cannnot be used to analyze intermittent spikes such as blobs, because it is intended for the periodic oscillation analysis. Thus, we introduced a probability density function (PDF) to analyze the time series signals acquired with the 2D-LiBP. PDF analysis has been applied to intermittent phenomena observed in various devices [16-18]. The PDF is a derivative of the probability distribution function (i.e., the rank function), generally, the PDF should be Gaussian when the signal is completely random. On the other hand, if the signal has more positive spikes than the negative ones, the PDF appears to be positively skewed, and vice versa.

Figures 3 (b) and (d) show PDFs derived from the emission signals of (a) ch1 and (c) ch7. The PDF in Fig. 3 (d) is almost Gaussian. In contrast, that in Fig. 3 (b) is non-Gaussian and positively skewed, indicating that the positive spikes dominate in the signal from ch1. To compare the PDFs of signals in various positions, the skewness of the PDF, which is the third moment normalized by the second moment around the mean value (i.e., the variance), is used for quantitative estimation.

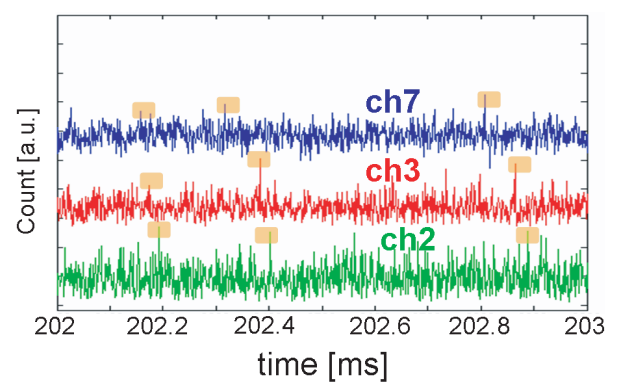

Fig. 2 Time series of Li I emission signals of ch2, ch3, and ch7. Some large spikes (marked with rectangles) seem to propagate from ch7 to ch2 through ch3.
The skewness $S$ is defined as

$$
S=\frac{\left[\int_{-\infty}^{\infty}(x-\mu)^{3} p(x) \mathrm{d} x\right]}{\left[\int_{-\infty}^{\infty}(x-\mu)^{2} p(x) \mathrm{d} x\right]^{3 / 2}},
$$

where $\mu$ is the mean value of the time series signal $x(t)$, and $p(x)$ is the PDF. For a PDF with a Gaussian shape, the skewness $S$ falls to 0 . If the pdf is positively skewed (i.e. positive spikes dominate), the skewness has a positive value.

Figure 3 (e) shows the 2D-profile of skewness $S$ in a discharge with $\bar{n}_{\mathrm{e}}=0.9 \times 10^{18}\left[\mathrm{~m}^{-3}\right]$. The skewness of fluctuation signals in all channels is positive in this discharge. $S$ tends to increase with distance from the confinement region. In other words, the skewness is positively large where the electron density is low in the ergodic region outside the confinement region.
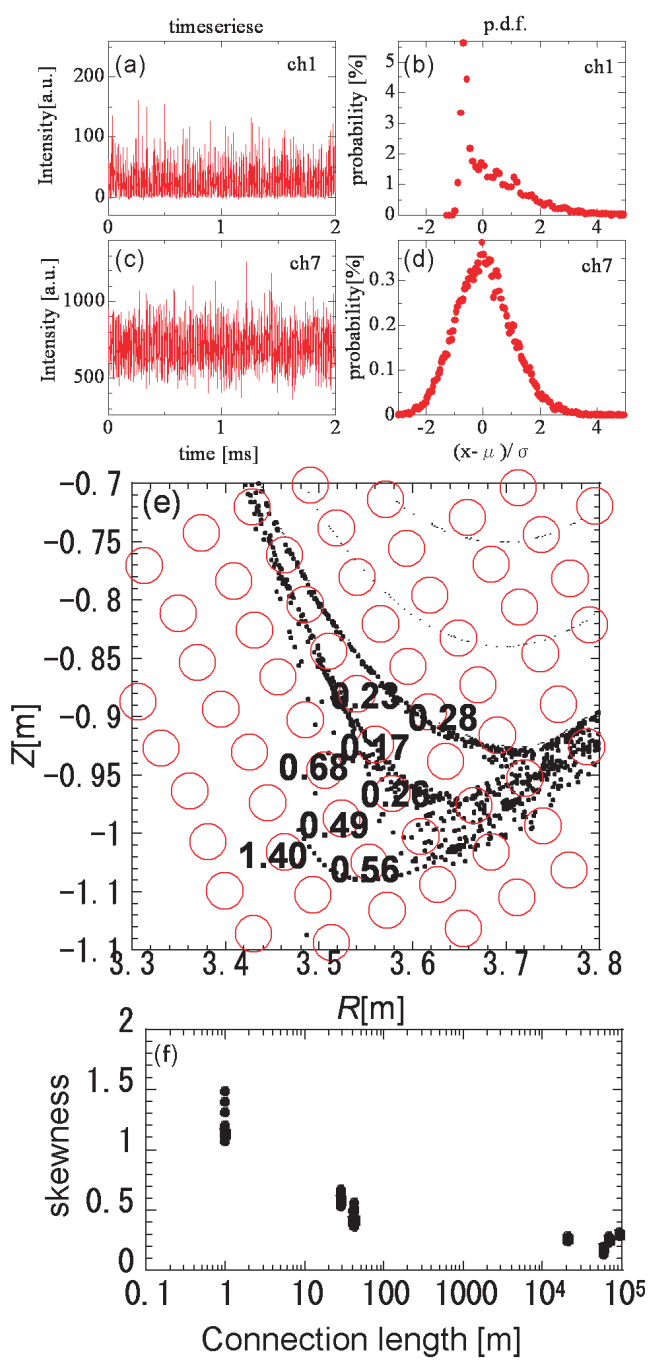

Fig. 3 Time series of Li I emission signals for (a) ch1 and (c) ch7, and the corresponding PDFs [(b) and (d), respectively]. Horizontal axis is normalized by standard deviation $\sigma$. (e) 2D -profile of skewness in the discharge with $\bar{n}_{\mathrm{e}}=0.9 \times 10^{18} \mathrm{~m}^{-3}$. (f) Relationship between skewness and connection length. 


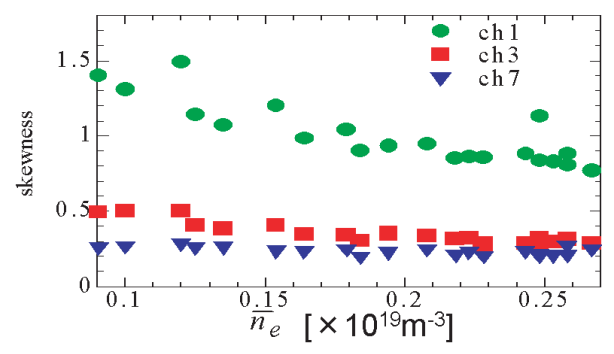

Fig. 4 Dependence of skewness on electron density in ch1, 3 and 7.

Table 1 Magnitude relation of skewness and fluctuation level.

\begin{tabular}{c|c|c|c} 
& edge & SOL & LCFS \\
\hline \hline skewness & Large & Small & Large \\
\hline fluctuation level & Large & Small & Large
\end{tabular}

To see the effect of the magnetic field structure on fluctuation properties, the relationship between the connection length of the magnetic field line $L_{\mathrm{c}}$ and the skewness $S$ was investigated, as shown in Fig. 3 (f). $S$ is small in the region where $L_{\mathrm{c}}$ is large. The plasma on the magnetic field lines with long $L_{\mathrm{c}}$ accumulates many fluctuations propagating in both parallel and perpendicular directions. Accordingly, the fluctuation there is turbulent. On the other hand, $S$ is high where $L_{\mathrm{c}}$ is small in the periphery of the ergodic region. In the heliotron configuration, the magnetic field lines in this region connect divertor plates in adjacent helical sections within a few meters. Therefore, plasma diffusing from the confinement region soon escapes to the divertor plates. Thus, only cross-field convective flux such as blobs can reach the ergodic region. With this assumption, it may be said that the fluctuations observed in this region are dominated by blobs.

As mentioned earlier, a relationship seems to exist between skewness and electron density: the skewness in the inner region with relatively high electron density is small compared to that in the outer region with low electron density. However, from the 2D -profile of skewness in Fig. 3 (e), it is difficult to distinguish the density effect from the magnetic structure effect discussed above.

To clearly distiguish them, it is necessary to see the effect of density on skewness at a fixed observing position. The dependence of skewness on line averaged density was obtained by a density scan experiment, as shown in Fig. 4. Three channels (ch-1, 3, and 7) aligned almost radially (see Fig. 1) were chosen for the calculation in Fig. 4. Clearly skewness tends to decrease with increasing in density, especially in ch-1.

Table 1 summarizes the tendency of the skewness and fluctuation profile, including additional discharge data where we can obtain data for the last closed flux surface (LCFS) and SOL regions simultaneously. These experimental observations suggest that blobs are born in the deep

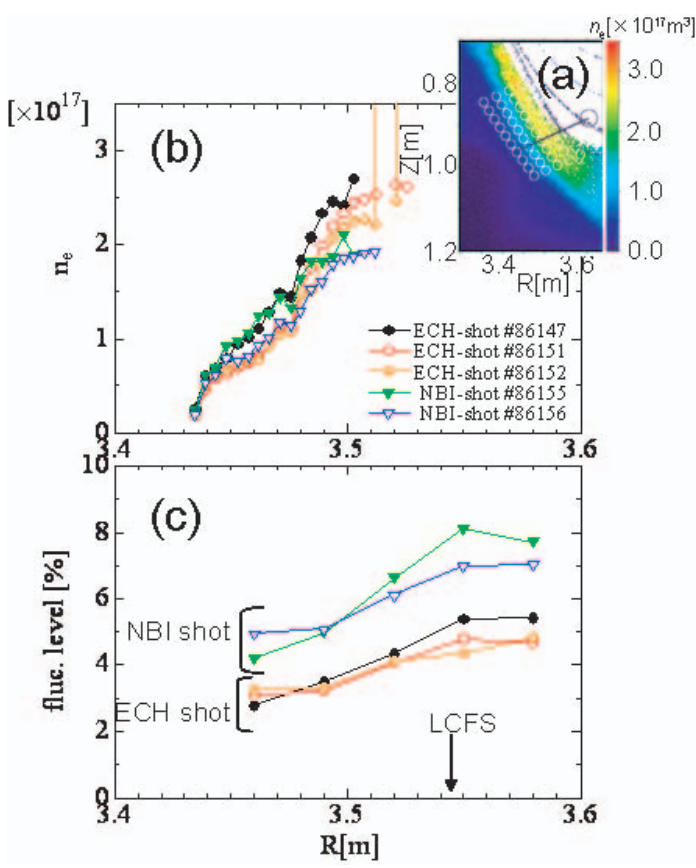

Fig. 5 (a) The electron density profile reconstructed from LiI emision, (b) the edge electron density profile along the solid line in fig. (a), (c) the fluctuation profile.

ergodic regionand then transported to the outer region with short $L_{\mathrm{C}}[18,19]$. It is also conceivable that the birth zone of blobs moves outward with increasing density.

The emission light is also measured by a CCD-camera (sampling rate: $10 \mathrm{~Hz}$ ) for density profile measurement. Figure 5 (a) shows the edge density profiles reconstructed from LiI emission. In the core region (white), the emission is very weak to allow reconstruction of the density profile because of the short penetration length. The density profiles along the solid line in Fig. 5 (a) are compared for neutral beam injection (NBI) discharge and electron cyclotoron heating (ECH) discharge [Fig. 5(b)]. Though there is no great difference in the edge density profiles, there is a significant difference in fluctuation level, as shown in Fig. 5 (c).

The properties of density fluctuations depend on the heating method. The skewness calculated from ch3 data for an NBI shot is larger than that of an ECH shot, even when the line-averaged density or electron temperature is almost the same. The increase in skewness is derived from the increase in the magnitude of positive spikes. The heating method is believed to influence the developmental mechanism.

Wavelet analysis is also suitable for time series signals of intermittent phenomena; thus, it has been used in many turbulence studies in plasma physics. We used this method to derive the effective blob velocity $V_{\mathrm{b}}^{\text {eff }}$, which is defined below, instead of using the time-of-flight method for individual blobs. Using the definition in Ref. [20], we computed a wavelet cross correlation function $C_{x 1, x 2}^{a}(\tau)$ between two signals, $x 1(t)$ and $x 2(t)$, at the wavelet scale 


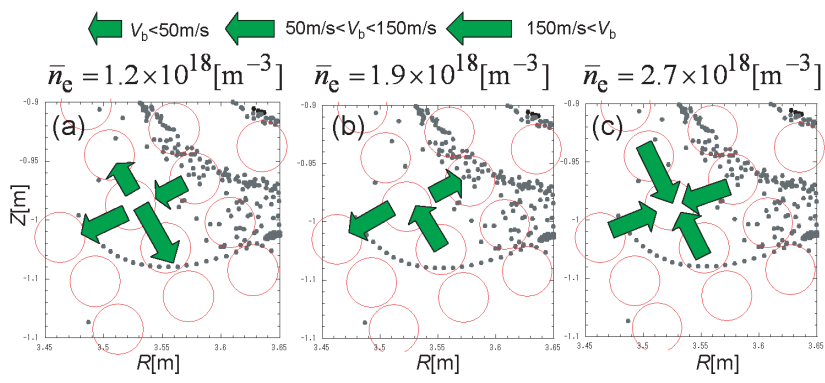

Fig. 6 Diagram of effective blob velocity at ch3. (a) $\bar{n}_{\mathrm{e}}=1.2 \times$ $10^{18} \mathrm{~m}^{-3}$ (b) $\bar{n}_{\mathrm{e}}=1.9 \times 10^{18} \mathrm{~m}^{-3}$ (c) $\bar{n}_{\mathrm{e}}=2.7 \times 10^{18} \mathrm{~m}^{-3}$.

$a$, where $\tau$ is the delay time between two signals. The phase delay $\tau_{a}$ of the wavelet cross correlation function, $C_{x 1, x 2}^{a}\left(\tau_{a}\right)=\max \left[C_{x 1, x 2}^{a}(\tau)\right]$, is used to calculate the velocity of a blob $V_{\mathrm{b}}^{a}$, which is defined by $V_{\mathrm{b}}^{a}=\Delta / \tau_{a}$ where $\Delta$ is the distance between two channels. $V_{\mathrm{b}}^{a}$ is calculated at the reference channel using any adjacent channel data. We found that blobs travel not only from the reference channel area to the adjacent channel area but also in the opposite direction. Since the direction and speed of each blob are different, the effective velocity of blobs $V_{\mathrm{b}}^{\text {eff }}$ is calculated, averaging $V_{\mathrm{b}}^{a}$ over the range $1 \mu \mathrm{s} \leq a \leq 100 \mu \mathrm{s}$ and $t_{0} \leq t<t_{0}+100 \mathrm{~ms}$ to minimize the statistical error. In the LiBP data, the number of spikes used for calculation is $350-400$. This number ensures the desirable satistical properties.

Figure 6 shows the results of the wavelet analyses. The block arrows represent the $V_{\mathrm{b}}^{\text {eff }}$ vectors for blobs entering or exiting reference ch3. The radial velocity is reported to be $2.6 \mathrm{~km} / \mathrm{s}$ and the poloidal velocity is $5.0 \mathrm{~km} / \mathrm{s}$ in a tokamak [2]. One of the blob velocities we observed in the LHD was $1.0 \mathrm{~km} / \mathrm{s}$, radially and $2.5 \mathrm{~km} / \mathrm{s}$, poloidally, which seems to show the same tendency for the ratio of radial velocity to poloidal velocity. The small effective velocity in Fig. 6 might indicate that the direction of propagation is nonunique. At low electron density (Fig. 6, $\bar{n}_{\mathrm{e}}=1.2 \times 10^{18} \mathrm{~m}^{-3}$ ), the blobs seem to move almost directly outward, which is same result often seen in the tokamak edge plasma. However, the transport characteristics change with increasing edge electron density, and the transport direction of the blobs becomes random. This kind of change has not been observed in tokamaks. This behavior may be attributed to edge differences in the magnetic field structures of tokamaks and heliotrons. If the radial transport of the blob is caused by $\nabla B$ and magnetic field curvature [1], its direction changes frequently due to helical ripples in the heliotron configuration. Thus, inward transport of blobs can occur in the heliotron configuration, unlike in tokamaks. Furthermore, in the edge region of the heliotron configuration, the $L_{\mathrm{c}}$ profile oscillates along the radial direction, while $L_{\mathrm{c}}$ in tokamaks decreases monotonically. It is possible for that the electric field in the heliotron configuration with oscillating $L_{\mathrm{c}}$, can change its direction according to the space potential on each magnetic field line. Therefore, if a blob originates in a slightly different position with a different electric field, its drift direction should be different. The interesting blob transport phenomena observed in the LHD may be due to characteristics of the heliotron configuration with its complicated edge magnetic field structure. Further experimental and theoretical studies are necessary to confirm this.

\section{Summary}

Iintermittent density oscillations, so called blobs, were observed with the 2D-LiBP in the LHD. It was clearly demonstrated that the skewness of blob signals depends on the connection length of the magnetic field lines and the electron density. The transport direction of blobs was also found to change with the density, suggesting a complicated mechanism of blob dynamics closely related to the edge magnetic field structure.

\section{Acknowledgment}

The authors sincerely thank Prof. N. Ohno for his useful discussions, and Prof. O. Motojima for the continuous encouragement. This work was funded by NIFS07ULPP507 and a Grant-Aid for Scientific Research from the Ministry of Educationm, Culture, Sports, Science and Technology (MEXT), Japan.

[1] S. I. Krasheninnikov, Phys. Lett. A 283, 368 (2001).

[2] J. A. Boedo, D. Rudakov, R. Moyer, S. Krasheninnikov, D. Whyte, G. McKee, G. Tynan, M. Schaffer, P. Stangeby, P. West, S. Allen, T. Evans, R. Fonck, E. Hollmann, A. Leonard, A. Mahdavi, G. Porter, M. Tillack and G. Antar, Phys. Plasmas 8, 4826 (2001).

[3] D. A. D'Ippolito, J. R. Myra and S. I. Krasheninnikov, Phys. Plasmas 9, 222 (2002).

[4] O. Grulke, J. L. Terry, B. LaBombard and S. J. Zweben, Phys. Plasmas 13, 012306 (2006).

[5] D. A. Russell, D. A. D’Ippolito, J. R. Myra, W. M. Nevins and X. Q. Xu, Phys. Rev. Lett. 93, 265001 (2004).

[6] V. Rozhansky, I. Senichenkov and I. Veselova, Phys. Plasmas 14, 052309 (2007).

[7] N. Nishino, Nucl. Fusion 46, S658 (2006).

[8] O. E. Garcia, N. H. Bian and W. Fundamenski, Phys. Plasmas 13, 082309 (2006).

[9] G. Q. Yu, S. I. Krasheninnikov and P. N. Guzdar, Phys. Plasmas 13, 042508 (2006).

[10] C. Hidalgo et al., C. R. Physique 7, 679 (2006).

[11] Y. Takahashi, T. Morisaki, K. Toi and LHD Experimental Group, J. Plasma Fusion Res. 1, 103 (2006).

[12] Y. Takahashi, T. Morisaki, K. Toi and LHD Experimental Group, J. Korean Phys. Soc. 49, S175 (2006).

[13] H. Tsuchiya, T. Morisaki, A. Komori and O. Motojima, Rev. Sci. Instrum. 77, 10F526 (2006).

[14] H. Tsuchiya, T. Morisaki, A. Komori, Y. Takahashi, K. Toi, H. Zushi, O. Motojima and LHD Experimental Group, Plasma Fusion Res. 2, S1096 (2007).

[15] O. Motojima et al., Nucl. Fusion 47, S668 (2007).

[16] G. Y. Antar, G. Counsell, Y. Yu, B. Labombard and P. Devynck, Phys. Plasmas 10, 419 (2003).

[17] H. Miyoshi, N. Ohno, S. Takamura, V. P. Budaev, 
Y. Uesugi, N. Asakura, Y. Miura, S. Masuzaki and A. Komori, Proceedings, 31st EPS Conference on Plasma Phys., London, 2004 Vol. 28G, P-5.101.

[18] N. Ohno, S. Masuzaki, H. Miyoshi, S. Takamura, V. P. Budaev, T. Morisaki, N. Ohyabu, A. Komori et al.,
Contrib. Plamsa Phys. 46, 692 (2006).

[19] T. A. Carter, Phys. Plasmas 13, 10701 (2006).

[20] V. P. Budaev, I. M. Pankratov, S. Takamura, N. Ohno, M. Takagi, H. Matsuno, M. Okamoto and S. K. Saha, Nucl. Fusion 46, S175 (2006). 